\title{
Lo ético y la «mera» lógica
}

\author{
FELIPE MARTÍNEZ MARZOA \\ Universidad de Barcelona
}

\begin{abstract}
RESUMEN. De la noción kantiana de «ética autónoma» es parte el reconocimiento de que cada decisión tiene la forma lógica de universal sin tener, en cambio, efectiva universalidad, por lo cual es posible que una decisión esté en contradicción con su propia forma lógica. Esto exige una averiguación sobre el significado de «contradicción» y de «mera» lógica en Kant con el fin de aclarar en qué sentido el enjuiciamiento moral ha de tener el carácter de confrontación «lógica».
\end{abstract}

\begin{abstract}
It belongs to Kant's notion of «autonomous ethics» to acknowledge that every decision has the logical form of a universal without having effective universality, which makes it possible for a decision to be in contradiction with its own logical form. This requires an investigation of the meaning of «contradiction» and "mere» logic in Kant in order to determine in which sense the moral judgement must have the character of a «logical» confrontation.
\end{abstract}

Nos proponemos ahora reconocer la interdependencia entre dos cuestiones interpretativas de carácter bastante general referentes, la una, a la Crítica de la razón práctica, la otra, a la Crítica de la razón pura. Ambas, cada una por separado o en contexto que abarcase las dos y en el que incluso estuviese implicada la interdependencia, han sido ya reiteradamente tratadas, pero no (y ése es ahora el intento) centrando la atención en el recíproco remitir de la una a la otra.

Términos como «ética autónoma» expresan el requerimiento de dar algún tipo de viabilidad a algo de lo que a la vez se asume (y por eso el problema tiene la entidad que tiene) que es una contradicción, a saber, que el criterio de enjuiciamiento ha de consistir en aquello mismo que es enjuiciado, esto es, en la decisión misma enjuiciada. El carácter de contradicción reside en que algo que no es sino la decisión misma en cuestión pueda a la vez ser transgredido por esa misma decisión ( $\mathrm{y}$, si no pudiese serlo, no podría ser criterio de enjuiciamiento) o, dicho de otra manera, que cada decisión haya de ser a la vez algo distinto (y eventualmente condenatorio) de ella misma (pues, si no lo fuese, no podría ser criterio de enjuiciamiento para ella misma). La fórmula de Kant para hacer inteligible esto incluye mostrar, por una parte, que la decisión tiene necesariamente la condición lógica de universal (es decir: de aplicable a un número infinito de casos 
posibles) y, por otra parte, que esa misma decisión no tiene efectiva universalidad, es decir, nunca se ejerce en un número infinito de casos, lo cual se debe a que, siendo «universal» en el sentido dicho, es, sin embargo, contingente. En esta situación, la contradicción es viable en ciertos casos, a saber, aquellos en los que ella esté vinculada a la universalidad lógica, o sea, en los casos de reglas de conducta que son contradictorias sólo por su condición de reglas, es decir, por su forma lógica de universales, no por la conducta que implican en cada caso individual; se puede mentir en todos y cada uno de los casos, y, sin embargo, no es posible pensar la mentira como algo que se hiciese bajo conjunto alguno de condiciones (es decir, en alguna universalidad lógica), o, dicho aun de otra manera, los papeles respectivos de regla y excepción no se reparten por la mera mayor o menor frecuencia, sino que dependen de ciertas condiciones lógicas; hay cosas que, siendo pensables en cualquier caso, sin embargo, sólo lo son como excepción, porque presuponen como regla lo contrario de ellas mismas. Decimos, pues, que la vía por la que se conduce la contradicción inherente al concepto de ética autónoma es el hallazgo kantiano de la decisión como algo que tiene la forma lógica de universalidad siendo, sin embargo, contingente. Tal hallazgo es, muy kantianamente, ni más ni menos que el reconocimiento del fenómeno mismo al que estamos llamando decisión, o sea, la delimitación de exactamente aquello que no es reductible a conocimiento, la cual conduce a que la misma decisión puede dar lugar, en diferentes situaciones materiales cognoscitivamente dadas, a conductas materiales (cognoscitivamente presentes) también distintas, estableciéndose así la noción de una función en la que tanto los elementos del conjunto de salida (situaciones dadas) como los del conjunto de llegada (conductas materiales) son objetos posibles de conocimiento, mientras que sólo la función misma (la «máxima») es la decisión, lo cual confiere a la decisión los caracteres simultáneos de universalidad como forma lógica y de contingencia (este último en el sentido de que cualquier decisión - cualquier máxima- es contingente, si bien siempre ha de haber alguna, una u otra $\mathrm{u}$ otra) ${ }^{1}$.

Si todo esto es así, entonces lo que hay como autoenjuiciamiento en las decisiones (es decir: como «ética autónoma») es ni más ni menos que la cuestión de compatibilidad con la forma lógica de universalidad, cuestión en efecto inevitablemente presente en cada decisión por el hecho de que cada una de ellas tenga, como decisión, la forma lógica de universalidad y a la vez pueda, en el sentido que ya hemos expuesto, no ser compatible con esa forma. Tal es el tipo de autoenjuiciamiento cuya inevitabilidad queda demostrada por la argumentación de Kant. Y la (in)compatibilidad con cierta forma lógica no puede ser de otro tipo que a su vez lógica, esto es, que la forma de universalidad que la decisión como tal tiene la convierta o no en

1 Cf. mi «Reconsideraciọ́n del concepto de ética autónoma», La ortiga, núm. 33-35, pp. 35-45. 
contradictoria. Tanto más por cuanto a la decisión no es inherente otra universalidad que la de forma lógica; no puede pertenecerle, por ejemplo, pretensión de universalidad efectiva, ya que ésta equivaldría a necesariedad, cuando por principio la máxima es contingente. De aquí que los famosos «pueda» o «puedas» de las fórmulas kantianas al respecto del tipo «que tu máxima pueda valer como» o «que puedas querer que» sólo puedan entenderse en el sentido de posibilidad lógica o ausencia de contradicción ${ }^{2}$.

Esta caracterización reclama sin duda algunas precisiones. Kantianamente no es lo mismo «contradictorio» que «imposible». Por ejemplo: «decaedro regular» 0 «triángulo biobtusángulo» son imposibles y, sin embargo, en sentido específicamente kantiano, de lo que se trata ahí no es de contradicción. En cambio, Leibniz considera contradictorios (y, por ello, imposibles, pues Leibniz sí hace equivaler lo uno a lo otro) los significados de esos sintagmas geométricos. No es que Leibniz y Kant discrepen en cuanto al decaedro regular o al triángulo biobtusángulo; quizá ni siquiera en cuanto a la noción de posibilidad; lo que sí es diferente en uno y otro de esos pensadores es lo que se entiende por «contradicción». El sentido kantiano de esto último es substancialmente más restrictivo, y lo que en él se entiende por «contradictoriedad» coincide con un tipo particular de imposibilidad, a saber, aquella que Kant considera basada en la «mera lógica», donde esta última expresión va con «mero concepto» y con «juicio analítico». Tiene su interés el tratar de ubicar en relación con todo esto la «posibilidad lógica o ausencia de contradicción» con la que nos hemos encontrado un poco más arriba a propósito de la compatibilidad con la forma lógica de universalidad en las máximas. Ahora bien, tal intento requiere una perspectiva algo más amplia.

En la tradición de la filosofía moderna hasta Kant, el territorio de la posibilidad y la imposibilidad es el de cierto tipo de verdades que en algunos momentos se llaman «necesarias» y que son un tipo permanentemente bien delimitado de verdades con independencia de si este adjetivo es o no el adecuado a cada interpretación de su especificidad. Lo que ahora nos importa al respecto es lo siguiente: todo el papel que esas verdades desempeñan en el tramo histórico citado presupone que las mismas están siendo tratadas como en efecto verdades, es decir: no como lo que vamos a llamar «enunciados semánticamente vacíos», entendiendo por tales aquellos tránsitos de sujeto a predicado en los que el tránsito mismo sólo tiene lugar como ficción gramatical, sin que en el contenido haya en efecto tránsito alguno. Es frecuente en la época que, cuando se quiere aludir a la vaciedad semántica tomada en este sentido, se mencione cierta «lógica» tardoescolástica, de cuyo caso se cree distinguir perfectamente el de las verdades pertenecientes al tipo del que nos

${ }^{2}$ En cuanto a la fórmula, también kantiana, de «la humanidad como medio» y «como fin», así como al verdadero estatuto de la discusión sobre la mentira, véase mi ya citado «Reconsideración...», op. cit. 
estamos ocupando, y ello incluso cuando ciertas tesis que pudieran parecer situadas en el nivel de aquella «lógica» adquieren en el nuevo contexto un sentido que las hace pertenecer al territorio de las verdades necesarias (véase lo que más abajo recordaremos a propósito de «A es $A »)$. Es este reconocimiento de la no vaciedad semántica lo que permite a Descartes hacer pasar el proceso de la «duda» por un momento en el que ella se refiere no ciertamente a alguna ni a cualquiera de las verdades del tipo representado por las matemáticas, pero sí al conjunto de todas ellas en el sentido de que quién sabe si la necesariedad de las mismas no pudiera serlo sólo como constitución de mi mente; y es esto mismo, con consecuencias en las que no es cosa de entrar aquí, lo que hay en la doctrina «de la creación de las verdades eternas». Como es sabido, no todas las fórmulas en las que la no vaciedad se expresa son compatibles con las que acabamos de citar, pero el supuesto mismo de la no vaciedad es común a «racionalistas» y «empiristas». En Leibniz es central el que, por de pronto, el propio «A es A» no es semánticamente vacío, pues significa que A no es algo del tipo de lo que hemos visto representado por «decaedro regular» o «triángulo biobtusángulo»; en efecto, no vale razonar por identidad a partir de expresiones como estas últimas ${ }^{3}$. Sobre esta base, la cuestión de en qué consiste la legitimación de un «A es A» llega a ser en Leibniz el problema de todos los problemas.

La suposición de no vaciedad semántica que acabamos de definir es, en el tramo histórico aludido, tan clara que ni siquiera es expresada. El primero que se plantea la cuestión es precisamente Kant. De ese planteamiento sale la confirmación expresa de lo que se venía dando por supuesto, es decir: no son enunciados semánticamente vacíos. Sólo que el hecho mismo de plantearse la cuestión comporta emplear en algún momento positivamente alguna noción que sea ni más ni menos que la de lo que aquí hemos llamado «enunciado semánticamente vacío», y esto conlleva formular en algún momento un «A es A» que se entienda de tal manera que sea precisamente aquello que no es el de Leibniz. Nuestro presente propósito requiere que hagamos algunas precisiones sobre el aparato conceptual que Kant monta al respecto.

Cualquier uso específicamente kantiano de la palabra «concepto» entiende por tal un quid que vale para una pluralidad, en principio infinita, de casos posibles; «gato» o «círculo» son conceptos porque hay infinitos posibles gatos e infinitos posibles círculos. Pues bien, cualquier concepto que lo sea en este sentido puede tomarse en al menos dos maneras: una de ellas es la de una suma de notas, la lista de condiciones cuyo cumplimiento por una

\footnotetext{
3 De hecho nuestros manuales de lógica niegan validez al modo silogístico que permitiría, razonando por identidad a partir de expresiones como las citadas, deducir que «algún decaedro es regular» o que «algún triángulo es biobtusángulo», pero ello se debe a que en nuestros manuales se asume que el «es» de la proposición particular tiene valor existencial y que, en cambio, el de la universal no tiene ni siquiera valor de posibilidad, mientras que Leibniz asume para todo «es» precisamente el valor de la possibilitas o essentia y ningún otro. La incongruencia, independientemente de la justificación que pueda tener, es nuestra, no de Leibniz.
} 
figura sea necesario y suficiente para que la llamemos «círculo» (algo así como que la distancia de cada punto a alguno fijo sea siempre la misma); la otra manera, en cambio, consiste en la regla que nos permite producir la figura de un ejemplar de la especie en cuestión, la de uno y luego la de otro y la de otro, empleando, sin embargo, siempre la misma regla, la regla para construir un círculo (por ejemplo: de un segmento de línea manténgase fijo uno de los extremos y muévase libremente el otro). La importancia de esta distinción puede sugerirse brevemente mediante una ilustración: si digo «tetraedro regular», «octaedro regular», «decaedro regular», estoy formulando conceptos como sumas de notas (a saber, en el primer caso, que tenga cuatro caras, que las caras sean iguales entre sí y sean polígonos regulares, etc.), y, mientras permanezca en este tipo de definición de los conceptos, no encuentro que en la de alguno de los tres mencionados ocurra nada que no ocurra en las de los otros dos; en cambio, si paso al intento de formular esos mismos conceptos en el modo de reglas de construcción de figura, entonces y sólo entonces descubro una abismal diferencia de estatuto dentro del trío de fórmulas, a saber, que «decaedro regular» no es nada, que no hay construcción para ese nombre; esto último es también lo que ocurre con «triángulo biobtusángulo», sólo que en este caso la frustración del intento de construcción es mucho más inmediata y trivial.

El concepto en cuanto usado meramente como suma de notas es llamado a veces por Kant el «mero» concepto, y ese uso de los conceptos es lo que él llama la «mera» lógica, llamando «juicios analíticos» a los enunciados en los que ese uso se expresa, es decir, a aquellos en los que el concepto sujeto es empleado sólo como suma de notas. Decíamos que el solo hecho de que la no vaciedad semántica de ciertos enunciados tenga que tematizarse (cuando antes se daba por supuesta) requiere que aparezca una noción en positivo de lo que nosotros más arriba hemos definido como «enunciado semánticamente vacío»; pues bien, esa noción es en Kant precisamente la de los «juicios analíticos». Y también decíamos que esto comportaría la aparición de un posible modo de decir «A es $A »$ en el que el significado de esta expresión no contendría ya la pretensión de que haya una possibilitas o essentia de A; esto es: un modo de hablar en el que pueda decirse que todo decaedro regular «es» decaedro y «es» regular; este modo de hablar es el uso del concepto como mera suma de notas (el «mero» concepto, la «mera» lógica, los juicios analíticos), ya que, en efecto, a ese nivel, «decaedro regular» tiene los mismos derechos que «tetraedro regular» y «octaedro regular». Por otra parte, todo esto obliga también a reservar algún término para designar aquella imposibilidad que es reconocible ya en ese nivel «meramente» lógico o de «mero» concepto o de juicio analítico, esto es, la que consista en a la vez inclusión y exclusión de una misma nota en una misma lista de notas; es entonces solamente este muy especial tipo de imposibilidad lo que se llama «contradicción», reserva terminológica que otros no tenían por qué hacer. 
Algunos cables han tenido que cruzarse para que el papel central de «A es A» en Leibniz haya llegado a malentenderse en el sentido de que entonces todos los juicios serían (en sentido kantiano) analíticos, cuando lo que ese papel significa es, para empezar, que el propio «A es A» no lo es ${ }^{4}$.

Como es sabido, el empleo kantiano de la expresión «mera lógica» para designar el proceder por «meros» conceptos o «juicio analítico» ha dado pie a que en fechas posteriores se asumiese que podría considerarse «juicio analítico» en algún sentido equivalente al kantiano, por lo tanto, con la connotación de lo que aquí hemos llamado «enunciado semánticamente vacío», sin valor de verdad en sí mismo, todo aquello que pudiese incorporarse a la lógica y ser considerado como «sólo lógica». El más conocido rendimiento de esta postura fue el proyecto del continuo lógico-matemático a conseguir por suma de la «logización de la aritmética» y la «aritmetización del análisis». Se pensó que, si ya no fuese posible establecer una frontera entre la lógica y las matemáticas, entonces los juicios de la matemática serían analíticos. El desarrollo de este proyecto fue fecundo en enseñanzas. Lo que aquí nos interesa es sólo lo que en el planteamiento mismo hay de malentendido a propósito del concepto de «mera» lógica: el proyecto, aunque sea contra Kant, pretende haberse apoyado en éste al asumir que la continuidad con la lógica comportaría carácter de juicio analítico, alegando que Kant mismo identifica los juicios analíticos con la «mera» lógica. Esto valdría sólo si lo que en esas fechas posteriores (en particular a partir de Frege) se entiende por «lógica», en definitiva, lo que hoy mismo entendemos por tal, fuese de alguna manera lo mismo (la misma noción, aun cuando hubiese experimentado desarrollos de uno u otro tipo) que aquello que en ciertos giros kantianos aparece designado como la «mera» lógica, o, dicho de otra manera, si nuestra lógica fuese en sentido kantiano «mera» lógica. Lo que impide cualquier asomo de respuesta afirmativa a esta cuestión no es el que una de las dos sea mucho más potente que la otra, sino el que entre esas dos cosas no hay ni siquiera el mínimo de base común para que la comparación sea posible. La cuestión de si los juicios de la matemática podrían ser analíticos (de «mera» lógica, basados en el «mero» concepto) no hubiera podido ni siquiera plantearse en serio si se hubiese atendido a qué es lo que han de significar las expresiones «mera lógica» y «mero concepto» para que con ellas tenga algún sentido definir una noción como la de «juicio analítico». No hay, en efecto, ni puede haber concepto alguno que la matemática pueda definir y con el que pueda operar en el modo de la mera suma de notas; y, por otra parte, este carácter de la matemática lo es también de la lógica misma en el sentido que la palabra «lógica» tiene comúnmente hoy. Ahora bien, todo esto no es sólo una cuestión de atribución de significado a términos más o

\footnotetext{
${ }^{4}$ Es verdad que ya el propio Kant entendió como «juicio analítico» ciertas cosas de Leibniz y de Hume que no lo son, pero ello está en relación con que Kant conocía sólo una pequeña parte de la obra de cada uno de esos dos pensadores.
} 
menos técnicos, como «mera lógica», «mero concepto» y «juicio analítico», sino que el hecho de que las nociones que kantianamente aparecen sean las que son responde a un requerimiento fenomenológico; algo hemos dicho ya de él, cuando lo hemos relacionado con la necesidad de tematizar el que, en cambio, ciertos otros enunciados no son semánticamente vacíos; pero vamos a ver que hay también, quizá paradójicamente, un necesario uso positivo de la noción de juicio analítico.

En el nivel de las meras definiciones nominales de términos, definimos «juicio analítico» en la manera que hemos dicho para a continuación llamar «sintético» a todo juicio que no responda a esa definición. Sin embargo, el desarrollo fenomenológico en el que estas definiciones tienen un papel pone de manifiesto que sólo hay en general concepto porque hay en todo caso síntesis y que ésta tiene que ver con lo que aquí ha aparecido como la regla de construcción, de manera que el «mero» concepto (y, por lo tanto, el juicio analítico) no es sino una secundaria «abstracción» en el sentido de que separa algo que de suyo no está por separado. El concepto tiene lugar como parte del fenómeno validez cognoscitiva, y lo que ahí tiene lugar es lo que hemos encontrado como la regla de construcción; de ésta resulta, ciertamente, un conjunto de condiciones, y por eso es segregable una lista de notas, bien entendido que esa segregación lo es con respecto al indivisible fenómeno conocimiento (validez cognoscitiva) y que por eso el empleo de «meros» conceptos (el juicio analítico) no es conocimiento. No por ello es menos cierto que hay, en efecto, la suma de notas, y que esto comporta la subsunción de unos conceptos bajo otros (subsumido es el concepto que tiene las mismas notas y alguna o algunas más). La subsunción no añade conocimiento alguno, y el orden que ella establece entre conocimientos no es el de la validez (fundamentación) de los mismos como tales; la gravitación no fundamenta la ley de la caída libre ni las órbitas de los planetas, sino que son estos dos conjuntos de fenómenos los que están en la fundamentación (empírica) de la gravitación, esto es, en su validación como conocimiento. El que este orden con el que ahora nos encontramos no sea el de la validez cognoscitiva responde, pues, a que estamos ahora ante un orden cuya necesidad estriba en que haya el «mero» concepto, el cual es precisamente el concepto segregado de su propio papel en lo que llamamos la validez cognoscitiva (segregado, es cierto, de su misma raíz). Por ello, si resultare que a ese proceso del subsumir son inherentes ciertas representaciones, éstas ya no tendrían la condición de algo inherente a que haya en general validez cognoscitiva, ya no pertenecerían a la possibilitas del conocimiento. Como es sabido, la importancia de esto radica en especial en que es por esa vía (la que aquí ha aparecido vinculada a la subsunción) como kantianamente se describe la naturaleza de las nociones de totalidad. Tal operación se realiza no sólo en relación con la validez cognoscitiva, sino también con lo que hemos llamado la decisión, o sea, la validez «práctica». También en este 
caso se considera entre unos y otros contenidos posibles de decisión un orden de dependencias que no lo es en cuanto a la fundamentación o validación de la decisión como tal, y también aquí, en consecuencia, el que a ese orden de dependencias sea inherente cierta representación de totalidad no convierte en manera alguna esa representación en integrante de decisión alguna. Para entender el que todo este modo de operar con las representaciones de totalidad tenga que ver en efecto con otros papeles del juicio analítico en Kant, tenemos ahora que introducir una consideración de índole más general.

El que la cuestión de en qué consiste la validez se mantenga distinta de cualquier cuestión de «el todo» de lo válido es rotunda peculiaridad de Kant dentro del pensamiento moderno. Tampoco Kant evita la noción de «el todo»; lo que hace es darle precisamente aquel carácter que impide que la validez pueda remitirse a ella. Esto tiene su coste. Recuérdese la referencia de Hegel al carácter «tautológico en el fondo» de la asunción de que esta mota de polvo sólo podría no tener lugar si «todo» cuanto hay (ha habido, habrá) tampoco tuviese lugar; lo que el argumento dice es que el ser de la mota de polvo no consiste sino en su estar avalada por «el todo»; la mota de polvo es contingente, por lo que en términos absolutos siempre cabrá seguir dudando de que, en efecto, la haya, no es posible un observador para el cual la haya sin posibilidad de duda en absoluto, y, por lo tanto, su «pero de suyo la hay» sólo puede querer decir que en ello se juega el ser de todo; el «de suyo» no significa sino el aval ejercido por «el todo». Esto resume el argumento básico del idealismo frente a Kant. Se admite (porque es tautológico incluso sin llegar al «fondo») que no hay certeza absoluta de facto, pero el solo hecho de admitir esto remite a algo así como una certeza absoluta de iure, un «sin embargo de suyo...», un in re, y no es posible atribuir significado a estas expresiones si no es por la vía de la vinculación con «el todo de lo ente», lo cual equivale a admitir un «ente uno todo». En consecuencia, el destierro kantiano de la noción de totalidad debe tener como precio la pérdida de la certeza absoluta incluso como mero de iure. Y, en efecto, así es; no sólo cualquier certeza que haya, sino la noción misma de certeza, la certeza de la cual se pregunta en qué consiste, es en Kant la certeza empírica. El idealismo esgrime frente a Kant el «principio realista».

Se pregunta - acabamos de decir - en qué consiste la certeza, donde «certeza» es la condición de certum, esto es, de definido y decidido, de precisamente así y no de otra manera, el discernimiento. Kantianamente la certeza es empírica, contingente sin paliativos, sin ni siquiera el recurso de la mota de polvo, puesto que la noción de «el todo» sólo es posible en términos tales que precisamente prohíben remitir a ella a la hora de decir en qué consiste la certeza. Es así tanto en lo cognoscitivo (validez cognoscitiva) como en lo práctico (decisión, validez práctica); un certum es un contenido de conocimiento (algo cognoscitivamente válido) o un contenido de deci- 
sión, y tanto lo uno como lo otro son empíricos con la connotación que acabamos de formular. En uno y otro caso lo que aparece como «necesario» es solamente lo constitutivo del «qué es» o «en qué consiste» (possibilitas o essentia) de la certeza misma, lo cual es necesario en el sentido de que, siendo en ello en lo que consiste que un contenido (siempre empírico) del tipo de certeza del que se trate (un certum del conocimiento o de la decisión) sea en efecto contenido (empírico) de ese modo de certeza (sea certum), ello es de antemano obligatorio para todo contenido de conocimiento (o, en su caso, para todo contenido de decisión). Y en uno y otro caso eso se reconoce constatando que hay el tipo de certeza en cuestión y describiendo (fenomenológicamente) eso que se constata, es decir: por una parte, constatando que hay validez cognoscitiva (lo cual no es reconocer contenido alguno como válido, sino que basta con encontrar la alternativa o la cuestión de válido y no válido) y describiendo en qué consiste; por otra parte, constatando que hay decisión (es decir, que la decisión no es reductible a conocimiento) y describiendo en qué consiste propiamente la decisión (es decir: qué es lo irreductible).

Motivo de típico reproche idealista es el que Kant trata los elementos constitutivos de la possibilitas de uno u otro tipo de certeza, es decir, eso necesario y a priori, de una manera en algún sentido empírica, como si fuese empírico; y, en efecto, no es empírico, pero sí fáctico, encontrado. Ello es consistente con el que los contenidos de la certeza (los certa) sí sean empíricos, ya que esto implica que la certeza misma como tal (la cuestión de si cierto o no cierto y de qué es cierto y qué no, en cada uno de los modos de certeza) es algo en lo que, simplemente, siempre ya se está. Y de que la certeza acontezca de este modo forma parte el que ni siquiera en el ámbito de la possibilitas de la certeza misma haya la unidad de un proceder deductivo, el que el propio «qué es» o «en qué consiste» de la certeza sea constatado, descrito, y, por lo tanto, que en él haya pluralidades no reductibles. En ello está incluido el que haya dos modos de validez o de certeza y que esa dualidad no sea ni deductible ni reductible, sino que sea encontrada. Ya se ha demostrado en otras ocasiones ${ }^{5}$ que la alusión a una «raíz común» se produce precisamente de manera tal que quede por completo excluido tanto el que eso «común» sea alguna validez o certeza, como el que de ello pudieran en algún sentido deducirse las «ramas».

Este carácter de facticidad que el ius mismo tiene (el que la validez, como cuestión de validez, sea algo con lo que nos encontramos), vinculado al carácter contingente de los contenidos (el «en qué consiste la validez» no genera contenidos), vinculado todo ello a la aparición de pluralidades no reductibles, es (todo ello junto) lo que llamamos la finitud característica de la posición de Kant. Con ella, pues, nos habíamos encontrado ya en las líne1992.

${ }_{5}^{5}$ Cf. mis libros Desconocida raíz común, Madrid, 1987, y De Kant a Hölderlin, Madrid, 
as iniciales del presente trabajo: encontrar algo inherente a la decisión como tal (encontrar que hay decisión y en qué consiste que la haya), describir el fenómeno que llamamos «decisión», equivalía a encontrar lo irreductible a conocimiento. A la luz de todo lo después dicho y de la posición en la que ahora estamos, el que hayamos ido a parar allí a algo que debía ser «lógico» (la compatibilidad con la forma lógica de universalidad) pudiera tener incluso un significado que allí no se hubiese explicitado todavía.

Para exponer el carácter de la decisión, habíamos recurrido en algún momento al concepto de una función en la que tanto los elementos del conjunto de partida como los del conjunto de llegada son objetos posibles de conocimiento, siendo la función misma lo único que no tiene ese carácter; en otras palabras: la máxima misma nunca está presente; lo presente son actuaciones materiales (cognoscitivamente observables) en situaciones también cognoscitivamente dadas, digamos: pares constituidos por un elemento del conjunto de partida y uno del de llegada. Lo único decidible es, pues, si la conducta material observable es tal que pudiera haber alguna máxima compatible con la forma lógica de universalidad (esto es, no contradictoria por el hecho de su propia forma lógica) y, a la vez, generadora de precisamente esa conducta. Esto es, en efecto, un examen crítico, pues, si no pudiese haber máxima alguna de esas condiciones, entonces yo ciertamente seguiría sin saber cuál es mi máxima, pero sabría que la misma, sea la que sea, es contradictoria con su propia forma lógica. En cambio, si encuentro que sí pudiera haber una máxima de las condiciones dichas, no por ello sé que mi máxima las cumple, puesto que sigo sin saber cuál es mi máxima. Ahora bien, la citada observación de incompatibilidad de cualquier máxima de cierto resultado con su misma forma lógica comporta, por de pronto, representarse con todas sus consecuencias necesarias la universalización de cierta conducta, no porque el adoptar ésta conlleve querer que ella se universalice, sino por lo que estamos diciendo de la confrontación con la forma lógica de universalidad. Y este representarse con todas las consecuencias necesarias es una operación de conocimiento; es conocimiento lo que nos dice que si..., entonces..., incluso en el caso de que la conexión sea necesaria en términos absolutos, pues en ese caso estaríamos ante lo a priori del conocimiento (que si son tres y se añaden dos, son cinco, etc.). Se trata de conocimiento, por lo tanto, no de juicios analíticos; pero es que eso todavía no es el discernimiento sobre compatibilidad o no con la forma lógica de universalidad; es sólo la presencia cognoscitiva de aquello (los elementos del conjunto de partida, los del conjunto de llegada) de lo que ya se ha dicho que, en efecto, es objeto posible de conocimiento. Una vez explicitado todo eso que es cognoscitivo, la regla de conducta conduce o no conduce a un «A no A», a un caso de «contradicción» en el restrictivo sentido kantiano arriba indicado, por lo tanto, de exclusión por juicio analítico o por «mera» lógica (en el sentido también ya expuesto). $Y$ tenía que ser así, porque en el paso mismo del 
enjuiciamiento de la posible máxima no puede haber ya nada que comporte conocimiento, pues, si lo hubiese, el enjuiciamiento ya no estaría en la decisión misma y no habría en absoluto «ética autónoma» ni irreductibilidad a conocimiento.

El que, una vez efectuada la representación cognoscitiva de la posible conducta, sea sólo contradicción de «mera» lógica (por lo tanto, el principio de lo «semánticamente vacío») lo que sirva para reconocer en su caso la incompatibilidad con la forma lógica de universalidad no convierte el enjuiciamiento en «vacío» él mismo, pues no es vacío el que haya esa exigencia de confrontabilidad con la forma lógica de universalidad. Lo único que la vaciedad en cuestión garantiza es el que en esa confrontación no haya ninguna otra cosa que precisamente esa confrontación. 